\title{
Regulation of a Distinctive Set of Genes in Glucocorticoid- evoked Apoptosis in CEM Human Lymphoid Cells
}

\author{
E. Brad Thompson and Betty H. Johnson \\ Department of Human Biological Chemistry and Genetics, The University of Texas \\ Medical Branch, Galveston, Texas 77555-0645
}

\begin{abstract}
Gene expression was evaluated in clones of the acute lymphoblastic leukemic cell line CEM that were sensitive or resistant to apoptosis evoked by the glucocorticoid, dexamethasone (Dex). Founding clones CEM-C7 (glucocorticoid sensitive) and CEM-C1 (glucocorticoid resistant) were subcloned to maximize uniformity of each population studied. Among subclones of $\mathrm{C} 1$, our original pseudodiploid clone of glucocorticoid-resistant cells, we found a high proportion of hyperploid clones. Most $\mathrm{C} 1$ subclones were glucocorticoid resistant but two $\mathrm{C} 1$ subclones were found to be revertants to glucocorticoid sensitivity. Glucocorticoid receptor content of the $\mathrm{C} 1$ subclones varied almost 5-fold but higher quantity of receptors did not guarantee steroid sensitivity. Gene expression analysis was carried out on microchips containing representations for $\approx 12,600$ human genes. When a group of four subclones from $\mathrm{C} 1$ (three glucocorticoid-resistant and one glucocorticoid-sensitive revertant) were compared with the glucocorticoid-sensitive subclone CEM-C7-14 for basal gene expression, the four $\mathrm{C} 1$ subclones clustered closely and far from C7-14. Thus, basal gene expression in the $\mathrm{C} 1$ subclones differed for a large number of genes from that in the $\mathrm{C} 7$ subclone. Reversion to glucocorticoid sensitivity did not cause a major shift in basal gene expression to a more C7-like state. Three clones (one revertant glucocorticoid sensitive from $\mathrm{C} 1$ subclone, one $\mathrm{C} 7$ sensitive subclone, and one $\mathrm{C} 1$ glucocorticoid-resistant subclone) were compared for the genes regulated by treatment for 20 hours with $10^{-6} \mathrm{M}$ Dex. This interval brings the cells to a point just before the onset of apoptosis. We tested the hypothesis that a distinctive set of genes would be regulated in the glucocorticoidsensitive clones. This proved to be so. In three experiments, at our chosen levels of discrimination, 39 genes were consistently induced $\geq 2.5$-fold and 21 genes were consistently reduced $\geq 2$-fold in glucocorticoid-sensitive clones but not in the glucocorticoid-resistant clone. The glucocorticoidresistant clone showed induction or reduction of 88 genes different from those regulated in the glucocorticoid-sensitive clones. These data support our hypothesis and further show that the glucocorticoid-resistant clone is capable of responding to steroid but with a different set of genes. We propose that a general metabolic switch accounts for the alteration.
\end{abstract}

\section{Introduction}

Glucocorticoids are a mainstay of therapy for many lymphomas and leukemias, due to the ability of such steroids to prevent the growth and to cause the apoptotic death of these malignant cells. While some effects of steroids may be 
derived from their ability to block or lower the production of various growth factors upon which lymphoid cells depend, malignant lymphoid cells frequently have achieved independence from exogenously produced factors. These cells either make and use the factors themselves in intracrine, autocrine, or paracrine pathways or become independent of them altogether. Glucocorticoids can act directly on such malignant cells to bring about apoptosis. Evidence that this is so originally came from mouse and human malignant cell lines (Baxter et al., 1971; Norman and Thompson, 1977) and subsequently was supported by studies of freshly isolated human leukemic cells (Kaspers et al., 1994).

The complete mechanism by which steroids bring about apoptotic death of leukemic lymphoid cells is unknown but several basic facts have been established, largely through the use of cultured cells. The first basic requirement for glucocorticoid-evoked apoptosis is a functional glucocorticoid receptor (GR). Cells containing nonfunctional GRs and those with very low normal GR content are invariably resistant. GRs are transcription factors, activated by their ligands, the steroids. Synthetic steroids, such as dexamethasone (Dex), frequently are used therapeutically in place of the natural human glucocorticoid, cortisol. In the absence of ligand, the GR is located primarily in the cytosolic compartment, in association with a complex of several other proteins (Housley et al., 1985; Sanchez et al., 1987; Pratt and Toft, 1997; Silverstein et al., 1999). When the ligand binds to its high-affinity site in the carboxy-terminal portion of the GR, the receptor presumably reconfigures, dissociates from its heteromeric binding partners, and translocates to the nucleus. There, in association with a variety of other transcription factors, coactivators, or corepressors, the GR acts to regulate the transcription of specific sets of genes (Ivarie and O'Farrell, 1978; O'Farrell and Ivarie, 1979; Young et al., 1981; Harrigan et al., 1989; Horwitz et al., 1996; McKenna et al., 1999; Holter et al., 2001).

Though necessary, the GR alone is not sufficient for producing apoptosis. Many leukemic cells contain plentiful quantities of normal GRs but are nevertheless strongly resistant to glucocorticoid-evoked apoptosis. In principle, these cells could fail to die in the presence of agonist glucocorticoids for many reasons. The steroid ligands could be blocked from passage through the plasma membrane, pumped rapidly from the cells, and destroyed metabolically or biochemically conjugated so that their function is blocked. Though some of those mechanisms may occur occasionally (Johnson et al., 1984), they seem to be used rarely in leukemic systems. A second theoretical group of mechanisms includes resistant cells that have genetically or phenotypically altered the response systems to glucocorticoids so as to resist their lethal effect. Among these possibilities are 1) loss or critical reduction in quantity of one or more factors with which the GR must interact, 2) development of a dominant-negative form of such a factor, or 3) improper posttranslational modifications of the GR or an interactive factor (Gruol et al., 1986). A third list of mechanistic possibilities 
involves changes that affect the general pathways for apoptosis: 1) alterations in the balance of pro- and antiapoptotic members of the Bcl2 family of proteins; 2) loss of or inactivating mutations in caspases or other lethal proteases; and 3) changes in one or more critical protease substrates, rendering it/them resistant. A final group of mechanisms could evoke alterations in specific genes' abilities to be regulated by ligand-driven GR: large-scale or selective heterochromatization or methylation of DNA (Gasson and Bourgeois, 1983; Gasson et al., 1983) or "insulation" of patches of genes could prevent the GR from properly affecting their transcription (Bell and Felsenfeld, 1999).

A second known basic requirement for GR-evoked apoptosis of lymphoid cells is cellular gene transcription and translation. In normal rodent thymocytes, as well as rodent and human leukemic cell lines, blocking cellular transcription or translation prevents the advent of the classic morphological and biochemical events in the apoptotic pathway (Wyllie et al., 1981,1984; Van den Bogert et al., 1988; Migliorati et al., 1994; Ramdas and Harmon, 1998; Mann et al., 2000). Although, given enough time, such inhibitors are themselves lethal, the tight correlations between the classic biochemical events preceding apoptosis and outright eventual cell death lead one to conclude that cellular macromolecular synthesis is necessary for glucocorticoid-evoked apoptosis. This is consistent with the fact that the GR is a transcription factor and also with the timing of cell kill. Unlike the rapid onset of apoptosis seen when the stimulatory agent simply causes activation of a caspase cascade (Pinkoski and Green, 1999), glucocorticoid-evoked apoptosis is initiated only after a delay. In freshly isolated mouse thymocytes, this delay is 1-2 hours, while in malignant human lymphoid lines, the time is much longer, requiring 24 hours or more (Thompson, 1999). Only after this delay interval do the markers accompanying apoptosis begin to be seen, followed later by the breakdown of cellular macromolecular synthetic pathways and outright cell death.

During the lag period, if the steroid is removed or replaced on the GR by an antagonist ligand, apoptosis does not occur. In other words, the agonist steroid must be continually present until the protease cascade is initiated and mitochondrial membrane disruption occurs with the consequent release of proteaseactivating factors (Thompson, 1999).

As to the precise genes whose expressions are altered in such a way to cause apoptosis, only a few landmarks have been identified. Several efforts to identify the critical genes by older technologies designed to locate mRNAs that differed in quantity after steroid treatment failed to identify the essential genes (Harrigan et al., 1989,1991; Briehl et al., 1990; Baughman et al., 1991; Caron-Leslie et al., 1991; Pearse et al., 1992; Chapman et al., 1995; Cidlowski et al., 1996). Consequently, the problem has remained unsolved and only recently has the advent of gene microarray technique permitted a search for the detailed data necessary to resolve the gene expression changes that lead to leukemic cell 
apoptosis. We have chosen to address this issue using clones of cells established from the CEM line.

\section{Clonal CEM Cell Lines as Models for Glucocorticoid-sensitive and Glucocorticoid-resistant Human Lymphoid Leukemia}

Most early studies of glucocorticoid-induced lymphoid cell death were carried out in rodents, either treating the whole animal with steroid or isolating thymocytes for in vitro treatment. Later, it was shown that some malignant lymphoid cells from these laboratory animals could be killed by corticosteroids (Baxter et al., 1971). Based on this knowledge, lines of cells such as S49 (Sibley and Tomkins, 1974; Sibley and Yamamoto, 1979), WEHI7 (Bourgeois and Newby, 1977), and P1798 (Thompson, 1991) were developed. Each of these and several other cell lines have proven very useful in studying glucocorticoid action.

It has long been recognized that glucocorticoids were effective as chemotherapeutic agents against several types of human leukemias and lymphomas but not against others. It also became obvious during the era of single-drug chemotherapy for leukemia that resistance often developed after treatment of the sensitive malignancies with glucocorticoids alone. How well the rodent models explained these observations in humans was dubious. In the early 1970s, the first few human cell lines of T-cell origin were established. Since, in general, primitive $\mathrm{T}$ cells (i.e., young thymocytes not yet differentiated so as to be only CD4 or CD8 surface antigen positive) were likely to be sensitive to glucocorticoid-evoked apoptosis, we screened the leukemic cell lines of T-cell derivation then available for sensitivity to cell death when exposed to Dex. This synthetic glucocorticoid agonist was chosen because of its high potency, use in therapy, and resistance to metabolic inactivation. Among the lines tested, we noted that CEM, derived from a 3-year-old female patient with late-stage acute lymphoblastic leukemia, was somewhat sensitive to Dex (Foley et al., 1965). Reasoning that the partial effect was likely due to the presence of a mixture of sensitive and resistant subpopulations, we immediately cloned the line and screened a number of clones for Dex sensitivity. As anticipated, these showed pure sensitivity or resistance. Two were chosen for detailed study: CEM-C7 as the prototypical sensitive clone, killed by GR-occupying concentrations of Dex or other agonist glucocorticoids, and clone CEM-C1 as the prototypical resistant cells, able to grow in up to $10^{-5} \mathrm{M}$ Dex, a concentration 100-fold greater than that required to fully saturate the GR (Norman and Thompson, 1977).

Many properties of the two clones were similar. Each was pseudodiploid, with a characteristic pericentric inversion of chromosome 9 and an extra chromosome 22 (Moore et al., 1985). They contained similar concentrations of GR (12,000-14,000 GR sites/cell, $\mathrm{Kd}=\approx 20 \mathrm{nM}$ Dex), showed the same weak ability to metabolize Dex, and both demonstrated induction of glutamine syn- 
thethase (Harmon et al., 1979; Harmon and Thompson, 1981,1982; Zawydiwski et al., 1983). The latter result suggested that the resistance of $\mathrm{C} 1$ cells to Dex-evoked apoptosis did not represent a global inability to respond to glucocorticoid but rather to some change that blocked the pathway to apoptosis. Both clones later were shown to be haploid for the normal GR $\alpha$ gene, with one normal and one mutant allele for the GR $\alpha$ gene. In both, the mutant allele contained a single amino acid substitution (Leu753Phe) in the ligand-binding domain (Ashraf and Thompson, 1993; Hillmann et al., 2000). This renders the GR produced from that allele incapable of retaining bound Dex under conditions that alter the receptor to its transcriptionally active form. Eventually, it was found that the Leu753Phe mutation had existed in the cells of the patient and was not a consequence of tissue culture conditions (Hillmann et al., 2000). Somatic cell hybrids were prepared between $\mathrm{C} 1$ cells and a completely Dex-resistant subclone of C7 (ICR-27) that had lost essentially all functional GR (GR-). The hybrids in mass culture and after cloning all were sensitive to Dex-evoked apoptosis (Yuh and Thompson, 1987). Restoring normal GR to the $\mathrm{GR}^{-}$subclone by transfection caused it to regain Dex sensitivity; thus the GR was both necessary and sufficient to restore the sensitive phenotype (Harbour et al., 1990). From the fact that the C1 $\times$ ICR-27 hybrid cells underwent apoptosis when exposed to Dex, we concluded that 1) the $\mathrm{C} 1$ cells supplied sufficient active GR to deliver the apoptotic signal from Dex in the hybrids and 2) $\mathrm{C} 1$ cells do not contain a dominant-negative function that blocks the apoptotic response. A later clue to the nature of the lesion in $\mathrm{C} 1$ cells came when we discovered that treating them with forskolin (to activate the cyclic AMP (cAMP) signaling pathway) restored Dex sensitivity (Medh et al., 1998). We believe that clones of the CEM line represent a useful model system in which to study the mechanism for glucocorticoidinduced leukemic lymphoid cell apoptosis.

The virtues and limitations of tissue-culture models for in vivo cellular behavior are well known but a few points bear repeating. By using a tissueculture system, we are able to study in detail the responses of a defined population of human leukemic cells to steroids and other agents. With this system, we can carry out experiments difficult or impossible to conduct in vivo. We can isolate the direct effects of the steroids on the leukemic cells, without the many complicating influences of secondary effects due to the actions of the agents on other cells and tissues. The use of clonal populations increases the uniformity of response and permits powerful genetic experiments. Obviously, the specifics of response uncovered in a particular clone of cells must be compared with other cells and in vivo systems before they can be considered to be generally applicable. Limits notwithstanding, the use of cultured cells - or better yet, clones of cultured cells - has provided enormously useful information about biochemical mechanisms. This has been true for systems from bacteria through yeast and higher eukaryotes. 


\section{Basic Responses of the Sensitive Clone CEM-C7 to Glucocorticoid}

When Dex (or another glucocorticoid agonist) is constantly present in the culture medium, the population-doubling time of the cells is maintained for at least 24 hours but, over several days afterward, increasing numbers of cells arrest in the G1/G0 phase of the cell cycle. Concomitantly, there is an increasing loss of clonogenicity (Harmon et al., 1979) and accumulative apoptosis. Time-lapse photomicrography of Dex-sensitive cells has shown that, following the 24-hour lag period, increasing numbers of cells change from their usual spheroidal shape to a collapsed, shrunken form. This appears to occur stochastically. For each cell, once the apoptotic process begins, the alteration events occur rapidly, beginning with violent membrane bubbling followed by shrinkage and collapse without immediate lysis (E.B. Thompson and B.H. Johnson, unpublished results). Over a longer period of hours, the collapsed cells lyse.

Biochemically, several major steps in the pre-apoptotic and apoptotic periods have been identified and are summarized in Figure 1. The earliest change in gene expression we have documented is a reduction in c-myc mRNA and protein, due to an inhibition of c-myc transcription (Zhou et al., 2000a). The reduction of mRNA levels begins as early as 1 hour after addition of Dex (Thulasi et al., 1993). Since the half-life of c-myc mRNA is $\approx 30$ minutes, the block of c-myc transcription must occur shortly after addition of Dex. The reductions are seen only in sensitive cells; resistant clones, whether or not they contain an active form of the GR, maintain normal cMyc levels in the presence of Dex. Interest-

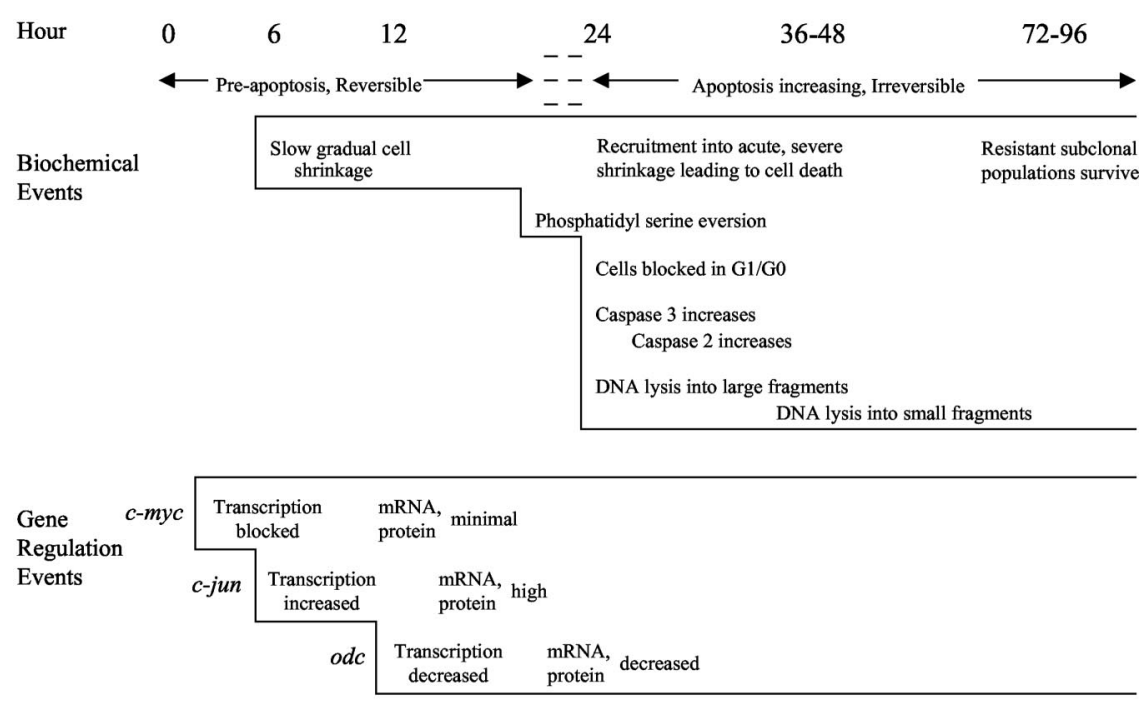

FIG. 1. Timing of some major events in CEM-C7 cells after addition of dexamethasone (Dex). 
ingly, when forskolin is used to stimulate the cAMP pathway in the $\mathrm{GR}^{+}$ resistant clone $\mathrm{C} 1$, addition of Dex kills the cells and cMyc is suppressed (Medh et al., 1998). Constitutive expression of ectopic c-myc delays glucocorticoidevoked apoptosis in C7 cells (Medh et al., 2001). The importance of c-myc downregulation in the death of lymphoid cells has been reviewed (Thompson, 1998).

Following in time, c-jun becomes chronically induced (Zhou et al., 2000b) and ornithine decarboxylase transcription is reduced (Miller et al., 2002). Eversion of phosphatidyl serine in the mitochondrial membrane begins $\approx 20$ hours after addition of Dex, while the first indications of increased caspase activity occur only after $\approx 24$ hours. DNA scission into very large fragments starts first after about 24 hours of Dex treatment and later after about 36 hours into $\approx 200$ base-pair units (Johnson et al., 1997). Both caspase activity and DNA lysis start slowly, then increase exponentially, consistent with the recruitment of increasing numbers of shrunken, apoptotic cells, noted microscopically. These cells eventually go on to lyse, leaving debris and some bits of DNA still contained within membranes (karyorrhexis).

\section{Quantity of GR Does Not Explain the Resistance of CEM-C1 Subclones to Glucocorticoid}

When the apoptosis-resistant clone $\mathrm{C} 1$ was originally established, careful comparison with the apoptosis-sensitive clone $\mathrm{C} 7$ indicated that, quantitatively and qualitatively, the GR content of the two was equivalent (Zawydiwski et al., 1983). Together with the somatic cell hybridization studies mentioned earlier, it was clear that lack of GR per se in C1 cells was not the explanation of their lack of apoptosis. Yet, the apoptosis-inducing function of the steroid-activated GR in $\mathrm{C} 1$ cells could be restored by forskolin treatment of the cells. Thus, it seemed that some interactive pathway was lacking. One report, using $\mathrm{C} 1$ cells presumably originally supplied from our laboratory, found that the population studied contained far fewer GR sites than did C7 cells. Transfection of these C1 cells with a plasmid designed to express normal GR $\alpha$ both raised cellular GR levels and rendered several subclones apoptosis sensitive to Dex (Geley et al., 1996). In considering this question, we suspected that the $\mathrm{C} 1$ cells employed in the study had undergone phenotypic drift and, as a consequence, had acquired a predominant population with low GR content. To test this possibility, we recloned our $\mathrm{C} 1$ cell population. Examination of the subclones showed a variety of karyotypes and GR phenotypes. The diversity of subclones we have subsequently uncovered within the $\mathrm{C} 1$ population would suggest that the cells in the above-cited study represented an overgrowth of subpopulations with the phenotype described. Some of these are listed in Table I. We noted a strong tendency for the $\mathrm{C} 1$ cells to develop hyperploidy, a phenomenon often seen in lymphoid malignancies 
TABLE I

Physiochemical Characteristics of CEM-C1 Clones

\begin{tabular}{llcccc}
\hline & $\begin{array}{l}\text { Resistant/ } \\
\text { sensitive }^{1}\end{array}$ & $\begin{array}{l}\text { Glucocorticoid } \\
\text { receptor sites/cell }\end{array}$ & $\mathrm{Kd}^{2}$ & Doubling time $^{3}$ & \% Hyperploid $^{4}$ \\
\hline CEM-C1 (1982) & Res & $10,000 \pm 700$ & $9 \pm 5.0$ & $25.7 \pm 1.8$ & 48 \\
Subclones $^{5}$ & & & & & \\
C1-15 & Res & $9,900 \pm 2,600$ & $12 \pm 3.2$ & $21.9 \pm 1.5$ & 0 \\
C1-11 & Res & $6,800 \pm 1,700$ & $7 \pm 0.2$ & $21.4 \pm 0.6$ & 0 \\
C1-4 & Res & $12,250 \pm 2,800$ & $8 \pm 2.3$ & $25.4 \pm 2.0$ & 84 \\
C1-16 & Res & $33,600 \pm 7,600$ & $9 \pm 0.4$ & $21.9 \pm 1.5$ & 72 \\
C1-6 & Sens & $27,200 \pm 3,200$ & $7 \pm 1.7$ & $25.4 \pm 2.9$ & 84 \\
C1-8 & Sens & $27,900 \pm 1,000$ & $7 \pm 0.2$ & $23.8 \pm 2.7$ & 72 \\
\hline
\end{tabular}

$\left[{ }^{1}\right.$ Resistant or sensitive to apoptosis in $10^{-6} \mathrm{M}$ Dex. ${ }^{2}$ Average number of Dex binding sites/cell and $\mathrm{Kd} \pm$ standard deviation (SD) from two to four multipoint Scatchard analyses. ${ }^{3}$ Average log-phase population-doubling time in hours $\pm \mathrm{SD}, \mathrm{n}=3 .{ }^{4}$ Average percent hyperploidy in 25 chromosome spreads on each of three separate slides. ${ }^{5}$ Subclones were obtained by the soft agar method (Harmon et al., 1985).]

(Secker-Walker, 1990; Pui and Crist, 1992; Martinez-Climent, 1997; Olah et al., 1997). In chromosome spreads of the uncloned $\mathrm{C} 1$ population, many hyperploid metaphases were noted. Among the clones we chose at random for karyotyping, $75 \%$ were subtetraploid. This is thought to arise because of nondisjunction at metaphase. Although it has been reported that, in general, hyperploidy correlates clinically with more treatment-sensitive behavior, ploidy in these clones did not generally correlate with resistance/sensitivity to glucocorticoids.

Low quantity of GR, as measured by specific, high-affinity cellular binding sites for Dex, did not consistently explain the resistance of $\mathrm{C} 1$ subclones to steroid-evoked apoptosis. GR quantity in the $\mathrm{C} 1$ clones totally resistant to $1 \mu \mathrm{M}$ Dex varied widely from 7000 to 33,500 GR sites/cell (Table I). Thus, the latter clone contained three times more GR than the sensitive clone C7-14, without showing Dex sensitivity.

Conceivably, the low-GR clones (e.g., C1-11, with $\approx 7000 \mathrm{GR}$ sites and relatively short doubling times) could overgrow the population under some conditions. Two subclones, C1-6 and C1-8, were found to have reverted to high sensitivity to Dex-evoked apoptosis. Both clones were hyperploid and contained higher levels of GR than the prototypical, pseudodiploid sensitive C7 clones. However, the fact that other hyperploid clones (e.g., C1-16) contained as much or more GR than clones C1-16 and C1-8, yet continued to be resistant, suggested 
that quantity of GR alone was not the explanation for the reversions. Gene-array analysis data (below) confirmed this suggestion.

The overall picture presented by the clonal CEM cell system, therefore, is that by activating the GR, Dex starts a process in which the ligand-activated receptor must participate continually for some 20-30 hours. This process culminates in activation of caspases (and possibly other proteases), with the consequence of relatively rapid and irreversible apoptosis. Resistance to this process cannot - in most cases - be explained by simple lack of GR. Consequently, we framed the following hypothesis: During the lag before overt apoptosis, an interactive network of genes undergoes altered expression. Some are controlled directly by the activated GR, others, indirectly, as a result of the changes in primary GR targets.

\section{Gene-array Analysis of Glucocorticoid Effects in Clones of Human Leukemic Cells}

We have begun to test the above hypothesis, employing several of these closely related clones of CEM cells.

\section{A. BASAL GENE EXPRESSION IN CEM-C1 SUBCLONES}

Gene-expression analysis was carried out by use of Affymetrix HG_U95Av2 chips, which carry sets of oligonucleotides capable of identifying $\approx 12,600$ human genes for which full-length cDNAs have been reported. The identity and functions of many of these genes are known. RNA samples were prepared from four of the $\mathrm{C} 1$ subclones, during midlog growth in RPMI 1640 medium supplemented with 5\% heat-inactivated fetal calf serum. Approximately half of the 12,600 genes were found to be expressed in the basal state in each of the clones. Cluster analysis showed that while the expressed gene sets of the four resistant clones generally were similar and quite different from sensitive clone C7-14, certain differences in expressed gene levels divided the four clones into two pairs (Figure 2). Clones C1-6 and C1-16 clustered together, as did the other pair, C1-15 and C1-12. C1-6 is Dex sensitive and C1-16 is resistant; therefore, their relative clustering in the basal state does not correlate with either phenotype. One obvious correlation that could be behind this clustering is that C1-6 and C1-16 are hypotetraploid clones, whereas the other clones are both pseudodiploid (Table I).

\section{B. COMPARISON OF APOPTOSIS-SENSITIVE WITH APOPTOSIS- RESISTANT CLONES}

Three of the freshly isolated clones were selected for closer analysis (Figure 3). C7-14 is a subclone of CEM-C7 and, like its parent, contains 11,000 GR 


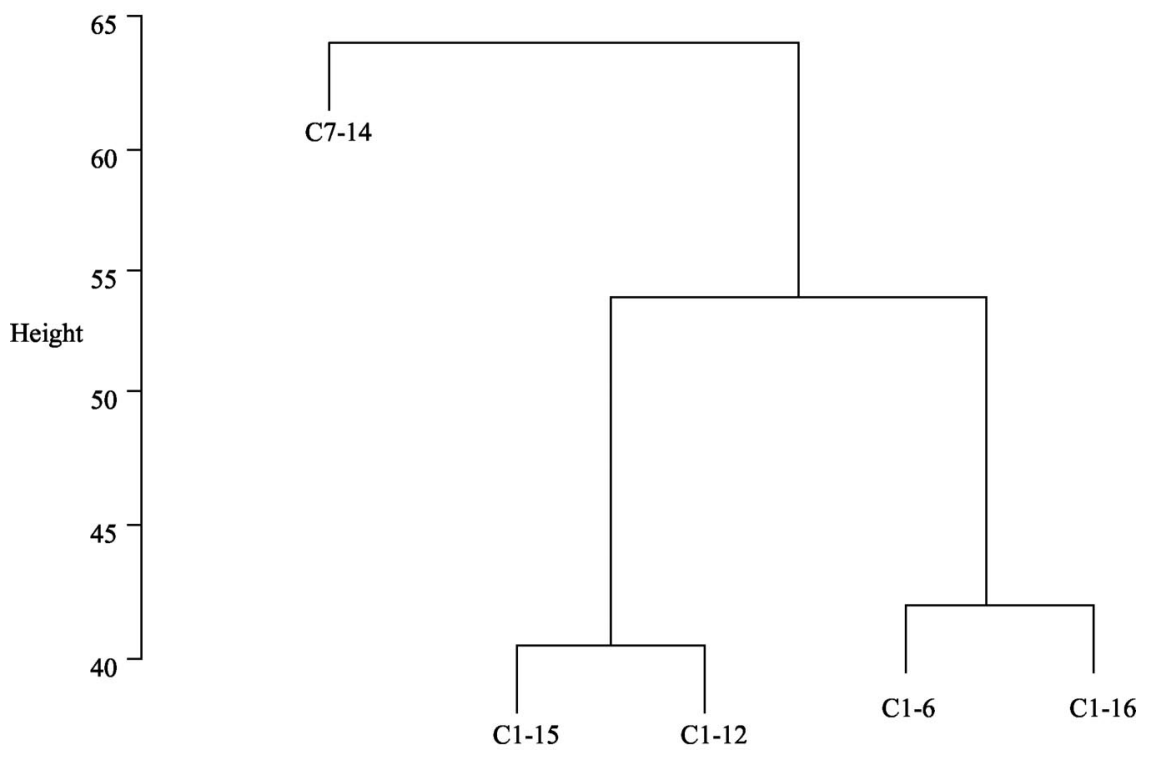

FIG. 2. Clustering of CEM cell clones by analysis of variance (ANOVA) of basal gene expression.

sites/cell and is sensitive to glucocorticoid-evoked apoptosis. C1-15 and C1-6 are subclones of $\mathrm{C} 1$. Clone $\mathrm{C} 1-15$ closely resembles the original properties of $\mathrm{C} 1$ in karyotype, strong Dex resistance, and GR content (statistically not different from C7-14). Clone C1-6 is interesting because it represents a rare revertant to sensitivity towards Dex-evoked apoptosis. In routine culture over its years of use, clone CEM-C1 never has been overgrown by a sensitive population and, among the clones chosen for study, only C1-6 and C1-8 showed high sensitivity.

Based on our original hypothesis as to the mechanism by which glucocorticoids cause apoptosis, we posed a subsidiary hypothesis, to be tested by gene-array analysis: A distinctive network of genes is regulated by glucocorticoids in the apoptosis-sensitive clones. These genes will not be regulated in the glucocorticoid-apoptosis-resistant cells. However, the functional GR of the cells resistant to glucocorticoid-evoked apoptosis still will be capable of regulating expression of some genes.

A time point of 20 hours following addition of Dex to the cultures was chosen, to be near the end of the lag period. In preliminary experiments, we confirmed that, after 20 hours in $10^{-7}$ M Dex, the sensitive C7-14 and C1-6 cells had not entered apoptosis (i.e., they still excluded propidium iodide vital dye). Between 24 and 48 hours, they showed increasing apoptosis, including overt cell death, just as the original CEM-C7 cells behaved. Clone C1-15 cells were 


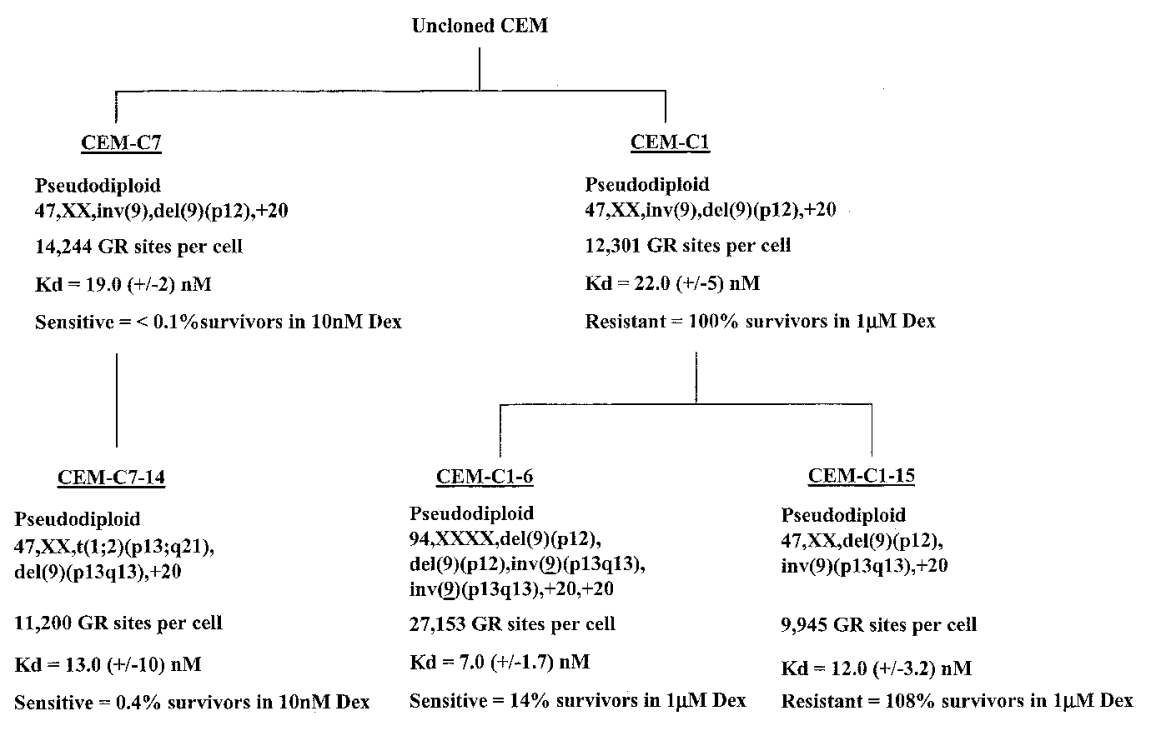

FIG. 3. Derivation of CEM clones.

completely Dex resistant in that they continued to grow at the same rate and showed no apoptosis to $1 \mu \mathrm{M}$ Dex over a period of 4 days.

For gene-array studies, the same protocol was carried out on three occasions over the course of a year. To cells in midlog growth, Dex was added to a final concentration of $10^{-6} \mathrm{M}$. Control cultures received an equal volume of the ethanol vehicle used to dissolve the Dex. Twenty hours later, the cells were collected and their RNA extracted. This late time point in the preapoptotic, reversible phase of Dex treatment was chosen to emphasize the accumulated changes in gene expression just preceding onset of full apoptosis. By repeating the experiment three times over a long period, we could test the constancy of the observed changes in gene expression and gain some statistical confidence of their relevance. Time-matched controls were included in each experiment. This approach is much safer than experiments involving a single sample at one time point and controls at only one time. The RNA was provided to the University of Texas Medical Branch Genomics Core Facility, where it was processed and analyzed by a single individual, using the methods prescribed by Affymetrix. The basic validity of the results was verified in several ways. We noted that, in the array results, as we and others had shown earlier by Northern blot analysis, c-myc mRNA consistently was reduced and GR $\alpha$ was induced. Second, we selected three genes that, according to the array data, were induced in the sensitive clones. Northern blots for those three mRNAs in cellular RNA extracts confirmed their clear induction (Table II). Third, Affymetrix chips often contain multiple probes 
TABLE II

Fold Induction by Dex ${ }^{1}$

\begin{tabular}{llccc}
\hline Gene & GenBank \# & C7-14 & C1-6 & C1-15 \\
\hline BTG-1 & (X61123) & 7.4 & 8.5 & 0.3 \\
DSCR1 & $(\mathrm{U} 85267)$ & 8.4 & 12.2 & 0.8 \\
NFIL3, E4BP4 & $(\mathrm{X64318)}$ & 21.1 & 7.9 & 0.4 \\
\hline
\end{tabular}

$\left[{ }^{1}\right.$ By image analysis of Northern blot.]

for the same gene at various locations on the chip. Several of the genes regulated in both sensitive clones appeared twice on our chips and, at both sites, gave comparable data and additional validation of the results. Induced genes were transforming growth factor beta (TGF $\beta$ ) receptor 2, interleukin (IL)-7 receptor, $\alpha$ tubulin, absent in melanoma 1 (AIM 1), inositol polyphosphate-1-phosphatase (INPP 1), and an unknown protein KIAA0878. The one repressed gene was c-myc.

For a first level of data analysis, we chose relatively stringent criteria for inclusion of a gene in the category "regulated by Dex." These criteria were greater than a 2.5 -fold increase or greater than a 2 -fold decrease in mRNA level in at least two of the three experiments. Validation studies in our Genomics Core Facility had shown that when a single RNA preparation was evaluated on three independent chips, less than $0.2 \%$ of the genes differed in expression by more than 2-fold. The higher limit of 2.5-fold was chosen to give an additional level of confidence that the changes seen were not due to random causes. On the other hand, we chose 2-fold reduction of mRNA level as the downward limit because, unlike fold induction, which theoretically can be infinite, fold reductions in mRNA level asymptotically approach zero. A 2-fold reduction in mRNA represents a 75\% reduction in the pool for that mRNA, a quantitative reduction likely to have functional significance.

When these criteria were applied, 39 genes were found to be induced greater than 2.5-fold in at least two of the three experiments in both C7-14 and C1-6 cells and were never or only once induced in C1-15 cells. A set of 21 genes distinctive to the two apoptosis-sensitive clones was also found to be repressed by 2 -fold (Table III). In these two gene sets, 22 genes were induced and four were repressed exclusively in both sensitive clones in all three experiments. When we examined the signal intensities for each of the genes that, in the three experiments, twice exceeded the chosen limits but once showed less than the cutoff regulation, the great majority in that experiment showed regulation in the correct direction, though quantitatively less than our chosen limits.

Examination of Table III also shows that other sets of genes were induced exclusively in clone C1-6 or C7-14, while a few others were induced in one of 
TABLE III

Distinctive Gene Sets That Are Dexamethasone Regulated in Clones of Cells Sensitive or Resistant to Glucocorticoid-induced Apoptosis

\begin{tabular}{|c|c|c|c|c|c|c|c|}
\hline & \multicolumn{3}{|c|}{ Single clone only } & \multicolumn{2}{|c|}{ Two clones } & \multicolumn{2}{|c|}{ All three clones } \\
\hline & C1-6 & C7-14 & C1-15 & $\begin{array}{l}\text { C1-6 + } \\
\text { C7-14 }\end{array}$ & $\begin{array}{l}\mathrm{C} 1-6+ \\
\mathrm{C} 1-15\end{array}$ & $\begin{array}{l}\mathrm{C} 7-14+ \\
\mathrm{C} 1-15\end{array}$ & $\begin{array}{l}\mathrm{C} 1-6+\mathrm{C} 7-14+ \\
\mathrm{C} 1-15\end{array}$ \\
\hline A & 71 & 47 & 62 & 39 & 3 & 3 & 5 \\
\hline B & 124 & 50 & 26 & 21 & 1 & 0 & 0 \\
\hline
\end{tabular}

[A $=$ number of mRNAs induced $>2.5$ fold in $\geq$ two of three experiments. $\mathrm{B}=$ number of mRNAs repressed $>2.0$ fold in $\geq$ two of three experiments. Each column gives the number of genes unique to that column (i.e., the 71 genes induced in C1-6 are uniquely so in C1-6, not in any other clones or combination).]

these clones as well as the apoptosis-resistant clone C1-15. Interestingly, clone C1-15 repeatedly showed unique induction of 62 genes. Similar patterns occurred for downregulated genes, though the numbers were smaller and, in several cases, zero. These data confirm our hypothesis that a distinctive set of genes is regulated in cells that show Dex-evoked apoptosis. Those that are found to be regulated in both sensitive clones are strong candidates for important players in the preapoptotic sequence. The specific sets of genes identified here are unlikely to include all those that are essential to glucocorticoid-evoked apoptosis. The chosen limits were deliberately made rather stringent and it is very likely that other genes, whose expression is altered to a lesser degree, will prove important to the process. For example, two genes that we know to be Dex regulated in CEM-C7 cells, c-jun and odc, fell outside our chosen limits.

Application of other statistical screening procedures (e.g., analysis of variance, ANOVA) can result in larger gene sets. The use of each particular statistical method has its own merits and limitations. For example, ANOVA evaluation of all three clones with a cutoff of $99 \%$ probability that any given gene was altered significantly differently from the rest identified some 350 genes regulated by Dex distinctive to the apoptosis-sensitive cells. ANOVA assumes that the populations considered follow a gaussian distribution. The fold-induction data actually do not do so. Thus, some of the genes accepted as induced (or deinduced) by ANOVA may be random variations. However, the conclusion that a unique set of genes is altered in the apoptosis-responsive clones is not changed. It should be recalled that the HG_U95Av2 gene chip only contains about one third of the genes expressed in human cells; hence, there are undoubtedly additional genes to be discovered that are relevant to this system. Unfortunately, many of the genes not found on this chip are only expressed sequence tags (ESTs), so much work will 
TABLE IV

Genes Induced or Deinduced by Dexamethasone in Both Apoptotic-sensitive Clones

\begin{tabular}{|c|c|}
\hline Induced gene & $\begin{array}{l}\text { Average-fold } \\
\text { change }\end{array}$ \\
\hline $\begin{array}{l}\text { CCR4, CC-CKR-4, K5-5, CMKBR4, CKR4, ChemR13, C-C chemokine } \\
\text { receptor-4 }\end{array}$ & 465.2 \\
\hline BIMEL, BCL2L11, BOD, BIM, BIML, BAM & 224.0 \\
\hline KIAA0620 protein & 215.0 \\
\hline Protein kinase $\mathrm{Ht} 31$ & 214.3 \\
\hline TUBA1, FLJ30169, alpha tubulin 1 & 112.8 \\
\hline TIP3, JAK, JAB, SOCS1, SOCS-1 & 34.4 \\
\hline BTG1, B-cell translocation gene 1 & 20.7 \\
\hline IL7R, CD127, interleukin-7 receptor & 12.1 \\
\hline AP1G2, G2AD, gamma2-adaptin & 11.4 \\
\hline SLC18A2, SVMT, VAT2, VMAT2, synaptic vesicle amine transporter & 7.4 \\
\hline Hypothetical protein MGC17330, clone DJ515N1 & 7.4 \\
\hline DKFZP586A0522 protein & 7.2 \\
\hline BIRC3, HAIP1, MIHC, HIAP1, API2, CIAP2, inhibitor of apoptosis protein 1 & 7.2 \\
\hline CD69, CD69 antigen & 7.1 \\
\hline DSCR1, MCIP1, ADAPT78, down syndrome candidate region 1 & 7.1 \\
\hline NFIL3A, NF-IL3A, IL3BP1, E4BP4, nuclear factor interleukin 3 & 6.8 \\
\hline seb4B, RNPC1, dJ800J21.2, HSRNASEB, SEB4D & 5.8 \\
\hline JAM3, FLJ14529 & 5.7 \\
\hline DFNA5, ICERE-1, deafness autosomal dominant 5 & 5.5 \\
\hline KIAA0386, C6orf32, DIFF40, DIFF48, PL48 & 4.8 \\
\hline KIAA0878 protein & 4.5 \\
\hline GR alpha, NR3C1, GRL, GR, GCR, glucocorticoid receptor & 4.2 \\
\hline TGFBR2, HNPCC6, transforming growth factor beta receptor II & 4.2 \\
\hline SLIM1, SLIMMER, FHL1, KYO-T & 4.1 \\
\hline AIM1, absent in melanoma 1 & 3.9 \\
\hline $\begin{array}{l}\text { PTK2B, PTK, FAK2, PKB, CADTK, CAKB, PYK2, RAFTK, cell adhesion } \\
\text { kinase beta }\end{array}$ & 3.5 \\
\hline TXNIP, VDUP1, THIF, EST01027, HHCPA78 & 3.4 \\
\hline $\begin{array}{l}\text { WFS1, WFRS, DFNA38, DFNA14, WFS, DFNA6, DIDMOAD, Wolfram } \\
\text { syndrome } 1\end{array}$ & 3.4 \\
\hline INPP1, inositol polyphosphate 1-phosphatase & 3.4 \\
\hline MAD-3, NFKBI, IKBA, NFKBIA & 3.1 \\
\hline KIAA0540 protein & 3.0 \\
\hline KIAA0920, paralemmin 2, A kinase (PRKA) anchor protein 2, AKAP-KL & 3.0 \\
\hline
\end{tabular}


TABLE IV

(continued)

\begin{tabular}{|c|c|}
\hline Induced gene & $\begin{array}{l}\text { Average-fold } \\
\text { change }\end{array}$ \\
\hline CD53 glycoprotein, MOX44 & 2.9 \\
\hline UQCRFS1, GRAP2 & 2.7 \\
\hline MAP1A, MAP1L, microtubule-associated protein 1A & 2.7 \\
\hline PRG1, PRG, hematopoetic proteoglycan core protein & 2.7 \\
\hline BTG2, TIS21, PC3 & 2.6 \\
\hline PTPRM, HR-PTPU, PTPRL1, RPTPM, protein tyrosine phosphatase receptor type M & 2.6 \\
\hline SLA1, SLAP, SLA, Src-like adapter protein & 2.5 \\
\hline Deinduced gene & $\begin{array}{l}\text { Average-fold } \\
\text { change }\end{array}$ \\
\hline RAD17 (S. pombe) homolog & 392.3 \\
\hline RAG1, recombination activating protein & 7.2 \\
\hline TLE4, transducin-like enhancer of split 4, homolog of Drosophila E(sp 1) & 6.7 \\
\hline $\begin{array}{l}\text { GPAT, PPAT, glutamine PRPP amidotransferase, phosphoribosyl pyrophosphate } \\
\text { amidotransferase }\end{array}$ & 4.5 \\
\hline Hypothetical protein MGC2574 & 4.0 \\
\hline HRY, hairy (Drosophila) A85 homolog & 3.2 \\
\hline HMGCS1, 3-hydroxy-3-methylglutaryl-Coenzyme A synthase 1 & 3.2 \\
\hline MYC, c-myc & 3.0 \\
\hline KIAA0830 protein & 2.7 \\
\hline DKFZP564C186 protein & 2.6 \\
\hline ITGA4, integrin alpha 4 (antigen CD49D, alpha 4 subunit of VLA-4 receptor) & 2.6 \\
\hline Protein predicted by clone 23733 , HSU79274 & 2.5 \\
\hline Hypothetical protein HSPC111 & 2.4 \\
\hline LTB, lymphotoxin beta, TNF superfamily member 3 & 2.4 \\
\hline $\begin{array}{l}\text { MEGF2, CELSR3, cadherin, EGF LAG seven-pass G-type receptor 3, flamingo } \\
\text { (Drosophila) homolog }\end{array}$ & 2.3 \\
\hline DKFZp586J0720 & 2.2 \\
\hline $\begin{array}{l}\text { PPP3CA, calcineurin A1, protein phosphatase } 3 \text { (formerly } 2 \mathrm{~B} \text { ) catalytic subunit- } \\
\text { alpha isoform (calcineurin A alpha) }\end{array}$ & 2.1 \\
\hline Hypothetical protein FLJ10534 & 2.1 \\
\hline PRMT3, protein arginine $\mathrm{N}$-methyltransferase 3 & 2.1 \\
\hline $\begin{array}{l}\text { DNA from chromosome 19-specific cosmid R30923, similar to RIKEN cDNA } \\
\text { 2410153K17 gene }\end{array}$ & 2.1 \\
\hline DTK, TYRO3, protein tyrosine kinase & 2.0 \\
\hline
\end{tabular}


be necessary to identify their full sequences, identities, and functions. Nonetheless, our initial, limited gene set already shows genes with some interesting properties, worthy of investigation for their relevance to the initiation of apoptosis.

Table III shows another interesting contrast between the two apoptosissensitive clones and $\mathrm{C} 1-15$, the resistant clone. Clone $\mathrm{C} 1-15$ also has a distinctive set of genes that are uniquely and consistently regulated by Dex. These data clearly indicate that there is a functional GR in C1-15 cells. One possible mechanism to explain the resistance of $\mathrm{C} 1-15$ cells is that a gene strongly protective against apoptosis is induced or that an antiapoptotic gene is deinduced. A search through the expressed gene arrays for the obvious candidates in these categories has not revealed any clear candidates. Thus, C1-15 cells contain an active GR that regulates many genes but these genes differ for the most part from those regulated by the GR in the clones that eventually undergo apoptosis after the addition of the GR agonist ligand, Dex.

Most of the regulated genes unique to clones C1-6 and C7-14 have been identified. Among these, several have shown antigrowth, proapoptotic, or prodifferentiation activities. Another group of genes was involved in various signal transduction or cell regulatory functions. The remainder fell into various groups, including a few structural genes. Few, if any, genes of general cell metabolism turned up in the set identified. Quite a few have received multiple working names or designations. These genes are listed by some of their names in Table IV.

These data demonstrate that the ligand-activated GR acts as both an inducer and a repressor of gene transcription. At least some of the regulated genes are controlled at the primary level by the GR. For example, inhibition of c-myc transcription is a primary effect of the activated GR, requiring no additional macromolecular synthesis (Medh et al., 1998; Zhou et al., 2000a). Not all of the genes need be regulated directly by the GR, however. The observed regulation of some genes is likely to be the consequence of GR-driven alterations in mRNA and protein levels of macromolecules that themselves serve regulatory functions. For example, we have shown that the GR-driven reduction in cMyc leads to a later reduction in ornithine decarboxylase, whose promoter contains a cMycbinding site. At that site, cMyc is a potent inducer of transcription. Thus, the loss of cMyc due to GR inhibition of c-myc transcription eventually results in a reduction of ornithine decarboxylase mRNA and protein. For other genes, GR-driven loss of a repressor of transcription could result in greater transcription of the gene secondarily regulated. In analogous fashion, GR-driven inductions could, by secondary or later sequential effects, produce either induction or repression of genes not under primary GR control. The GR itself could participate in these derivative regulations, for genes where the GR acts only in a heteromeric complex with other transcription factors. 
Hypothesis for the underlying mechanism of resistance or sensitivity to glucocorticoid-evoked apoptosis: We propose that the critical difference between the sensitive and resistant clones is in the nature of a switch, one that shifts the sets of responsive genes from one group to another. One group of regulated genes culminates in turning on apoptosis, while the other does not. The nature of the proposed switch is unknown; it could be genetic, epigentic, or purely regulatory.

\section{Comparisons with Earlier Studies of Related Systems}

In a previous attempt to identify genes relevant to glucocorticoid-evoked apoptosis in CEM cells, the effect of Dex on growth-arrested compared to growing cells was studied (Tonko et al., 2001). A growing CEM-C7 subclone (CCRF-CEM-C7H2) was exposed to $10^{-7} \mathrm{M}$ Dex and single samples were taken for analysis at 0,2 , and 8 hours. Against this were compared results from CCRF-CEM-C7H2-GE2-p16 cells treated with doxycycline to cause growth arrest. These cells had been prepared from the $\mathrm{H} 2$ clone by expressing in them the cyclin-dependent kinase inhibitor p16 (INK4A) gene, causing G0/G1 arrest. This had been found to heighten their sensitivity to Dex-evoked apoptosis (Ausserlechner et al., 2001). The primary array analysis was carried out by a commercial concern and the data subsequently analyzed by the authors. Despite its many limitations (e.g., one set of data at each time point, no time-matched controls, no evaluation of the effects on gene expression of doxycycline itself or of the introduction of the INK4A gene, and no comparison of the same cells (i.e. the INK4A-expressing cells, treated by Dex when growing and growth arrested)), the paper does show that a limited number of genes are altered by Dex treatment. The authors found that only eight genes were altered beyond the chosen limits, in both the growth-arrested and growing cells, at the times chosen for comparison. It is not made clear why one should expect the same genes to be altered in the growing cells many hours before their growth arrest as those in previously growth-arrested cells. We found none of these eight genes to be altered in our clones under our experimental conditions.

A second study from the same laboratory compared the effects of Dex treatment on CEM-C7 cells with those on another human leukemic line, Jurkat (Obexer et al., 2001). The Jurkat cells were of two sorts, one (clone A11-1) transfected so as to stably express a high level of wild-type (WT) rat GR, the other transfected with the GR-LS7 mutant gene (clone F6-1). The mutant GR (Miesfeld et al., 1987; Helmberg et al., 1995) was about half as potent overall in transactivating genes as WT GR. Both the WT and the mutant GR repressed genes overall to about the same extent. An early-version Affymetrix chip Hu6800/HuGeneFL containing probes for about 5600 genes was employed. Samples were taken at 0,3 , and 8 hours after addition of Dex. Generous criteria were chosen for accepting an expression change as valid, making it difficult to be 
sure whether some of the changes seen are due to random variations. This problem is compounded by analyzing only one experiment per time point. Nevertheless, several genes were found to be regulated similarly at one or more time points in the CEM and Jurkat cells.

Our data were obtained at a much later time, close to when apoptosis begins in CEM cells. We sought to determine the culminating gene changes that bring on that crisis. We employed Affymetrix chips that contain probes for $\approx 12,600$ genes, about twice the number found on the chips in the earlier studies. We also carried out three independent experiments in order to have greater confidence that the changes observed were not due to random events. Indeed, we observed that many genes appeared to be altered in expression in only one of the three experiments. This was true for both the apoptosis-resistant and apoptosissensitive clones.

When we compared the list of genes consistently altered after 20 hours of Dex in our CEM clone set with those found at the earlier time points in other CEM or Jurkat cells examined by Tonko et al. (2001) and Obexer et al. (2001), we found some concordances. A few genes observed to be altered by $\geq 2$-fold at 3 or 8 hours were also in our 20-hour set. Others found in our set had shown a lesser degree of altered expression in the earlier data from the other CEM-C7 subclones or in the Jurkat cells. The set of genes we found to be distinctively altered in the late preapoptotic stage did not provide much support for the theory of metabolic pathway alterations proposed earlier (Tonko et al., 2001).

Concordance between certain of the genes we detected and those found earlier in single-datapoint experiments provides added confidence that they may be universally important in lymphoid leukemic cell apoptosis. Several possibilities exist regarding the genes that do not match in these "discovery" experiments. The most exciting is based on our hypothesis that the steroid evokes a sequential network of genes: genes induced or repressed directly by activated GR, genes altered as a consequence of those direct effects, and reverberations stemming from these changes, including, for example, genes whose expression is altered as a result of posttranslational effects caused by the protein products of initial gene changes. An example might be an induced protein kinase whose function is to activate or deactivate by phosphorylating some gene-specific transcription factor; another would be proteins that reconfigure chromatin. Many other examples, of course, can be listed. To evaluate this time-dependent sequential gene network theory properly, we are undertaking a full kinetic analysis of our clone set.

Other more-trivial reasons may explain the lack of concordance of some genes in the data from the several experiments in two different laboratories. Some of the genes that seem to be altered in single experiments may not be due to the steroid but only be random variations in expression. Some may be clone specific and not universally important for glucocorticoid-evoked apoptosis. In 
some cases, the methods employed may have lacked sufficient sensitivity. Since our later experiments used chips containing twice as many genes, we may have discovered some responding genes not represented on the earlier chips. After all these explanations are considered, the fact remains that the concordance of a number of gene expression alterations due to Dex in several similar clones of all cells originally grown from two different patients is consistent with our sequential gene network hypothesis.

\section{Summary}

We have applied microchip gene-array analysis to the problems of phenotypic variability in cultured cells and glucocorticoid-induced apoptosis of lymphoid cells. The system used was closely related clones of human acute lymphoblastic leukemia cells, all derived from the CEM cell line. To study the problem of phenotypic variability, so common in long-term cell culture, we compared subclones derived from our two original CEM clones: CEM-C7 that undergoes apoptosis in glucocorticoid and CEM-C1 that is resistant. The subclones generally maintained the gross initial parental phenotype but showed frequent conversion to polyploidy. Gene arrays showed that the basal gene expression set of the subclones derived from the CEM-C1 (resistant) parent resembled one another more than they did the CEM-C7 (sensitive) parent or its subclones. Within the resistant subclones, the hyperploid subclones (regardless of their Dex resistance or sensitivity) resembled each other more than they did the pseudoploid subclones. Individual clones of the same gross phenotype showed considerable overlap in gene expression pattern but did differ with respect to some genes.

To analyze the difference between cells that respond to glucocorticoid by undergoing apoptosis and those that are resistant, we compared three clones. One clone was resistant to apoptosis in the presence of the glucocorticoid Dex. The other two were sensitive; one was a revertant from the CEM-C1-resistant clone. In the constant presence of a Dex concentration that fully occupied the GR, the two sensitive clones, after a delay of at least 24 hours, increasingly underwent apoptosis. We tested the hypothesis that the sensitive clones would show regulation of a distinctive set of genes, prior to the onset of actual apoptosis. Dex or ethanol vehicle was added to each clone and, 20 hours later (a few hours before apoptosis), the cultures were collected, RNA extracted, and mRNA levels examined on Affymetrix HG_U95Av2 chips. After three such experiments, we asked for genes that were consistently induced or reduced in the two sensitive but not the resistant clone. The results supported our hypothesis. Within our chosen limits, a group of 39 genes was consistently induced and one of 21 consistently deinduced only in the apoptosis-sensitive clones. A different set containing 88 genes was regulated consistently in the resistant clone. 
Comparing our results with two earlier reports containing data from single experiments on similar cells, but at earlier time points, discovered some concordances in the genes expressed. Many of our discovered genes were not seen as altered in the earlier experiments. The potential reasons are discussed. Overall, the results are consistent with our hypothesis of a sequential network of altered gene expression (both induced and repressed) being responsible for the ultimate apoptosis in the sensitive cells.

Our data also clearly show that the resistant clone responds to Dex by induction or repression of a significant number of genes. Therefore, the resistance is not due to lack of activated GR but to an alteration in the gene responses to GR. We propose that this is caused by an as-yet-undiscovered switch mechanism that converts sensitive to resistant.

\section{ACKNOWLEDGMENTS}

The authors would like to recognize the work of Scott Webb and Bruce Luxon for data analysis and both Scott Webb and Aaron Miller in this laboratory for the preparation of figures and graphs.

\section{REFERENCES}

Ashraf J, Thompson EB 1993 Identification of the activation-labile gene: a single point mutation in the human glucocorticoid receptor presents as two distinct phenotypes. Mol Endocrinol 7:631-642

Ausserlechner MJ, Obexer P, Wiegers GJ, Hartmann BL, Geley S, Kofler R 2001 The cell cycle inhibitor p16(INK4A) sensitizes lymphoblastic leukemia cells to apoptosis by physiologic glucocorticoid levels. J Biol Chem 276(14):10984-10989

Baughman G, Harrigan MT, Campbell NF, Nurrish SJ, Bourgeois S 1991 Isolation and characterization of glucocorticoid- and cyclic AMP-induced genes in T lymphocytes. Mol Cel Biol 9:3438-3446

Baxter JD, Harris AW, Tompkins GM, Cohn M 1971 Glucocorticoid receptors in lymphoma cells in culture; relationship to glucocorticoid killing activity. Science 171(967):189-191

Bell AC, Felsenfeld G 1999 Stopped at the border: boundaries and insulators. Curr Opin Genet Dev 9(2):191-198

Bourgeois S, Newby RF 1977 Diploid and haploid states of the glucocorticoid receptor gene of mouse lymphoid cell lines. Cell 11:423-430

Briehl MM, Flomerfelt FA, Wu XP, Miesfeld RL 1990 Transcriptional analyses of steroidregulated gene networks. Mol Endocrinol 4:287-294

Caron-Leslie LM, Schwartzman RA, Gaido ML, Compton MM, Cidlowski JA 1991 Identification and characterization of glucocorticoid-regulated nuclease(s) in lymphoid cells undergoing apoptosis. J Steroid Biochem Mol Biol 40(4-6):661-671

Chapman MS, Qu N, Pascoe S, Chen WX, Apostol C, Gordon D, Miesfeld RL 1995 Isolation of differentially expressed sequence tags from steroid-responsive cells using mRNA differential display. Mol Cell Endocrinol 108(1-2):R1-R7

Cidlowski JA, King KL, Evans-Storms RB, Montague JW, Bortner CD, Hughes FM Jr 1996 The biochemistry and molecular biology of glucocorticoid-induced apoptosis in the immune system. Recent Prog Horm Res 51:457-490 
Foley GR, Lazart H, Vzman S, Bonne BA, McCarthy RE 1965 Continuous culture of human lymphoblasts from peripheral blood of a child with acute leukemia. Cancer Res 18:522-529

Gasson JC, Bourgeois S 1983 A new determinant of glucocorticoid sensitivity in lymphoid lines. J Cell Biol 96:409-415

Gasson JC, Ryden T, Bourgeois S 1983 Role of de novo DNA methylation in the glucocorticoid resistance of a T-lymphoid cell line. Nature 302:621-623

Geley S, Hartmann BL, Hala M, Strasser-Wozak EM, Kapelari K, Kofler R 1996 Resistance to glucocorticoid-induced apoptosis in human T-cell acute lymphoblastic leukemia CEM-C1 cells is due to insufficient glucocorticoid receptor expression. Cancer Res 56(21):5033-5038

Gruol DJ, Ashby MN, Campbell NF, Bourgeois S 1986 Isolation of new types of dexamethasoneresistant variants from c cAMP-resistant lymphoma. J Steroid Biochem 24(1):255-258

Harmon JM, Thompson EB 1981 Isolation and characterization of dexamethasone-resistant mutants from human lymphoid cell line CEM-C7. Mol Cell Biol 1:512-521

Harmon JM, Thompson EB 1982 Glutamine synthetase induction by glucocorticoids in the glucocorticoid-sensitive human leukemic cell line CEM-C7. J Cell Physiol 110:155-160

Harmon JM, Norman MR, Fowlkes BJ, Thompson EB 1979 Dexamethasone induces irreversible G1 arrest and death of a human lymphoid cell line. J Cell Physiol 98:267-278

Harbour DV, Chambon P, Thompson EB 1990 Steroid mediated lysis of lymphoblasts requires the DNA binding region of the steroid hormone receptor. J Steroid Biochem 35:1-9

Harrigan MT, Baughman G, Campbell NF, Bourgeois S 1989 Isolation and characterization of glucocorticoid- and cyclic AMP-induced genes in T lymphoid. Mol Cell Biol 9:3438-3446

Harrigan MT, Campbell NF, Bourgeois S 1991 Identification of a gene induced by glucocorticoids in murine T-cells: a potential G protein-coupled receptor. Mol Endocrinol 5:1331-1338

Helmberg A, Auphan N, Caelles C, Karin M 1995 Glucocorticoid-induced apoptosis of human leukemic cells is caused by the repressive function of the glucocorticoid receptor. EMBO J 14(3):452-460

Hillmann AG, Ramdas J, Multanen K, Norman MR, Harmon JM 2000 Glucocorticoid receptor gene mutations in leukemic cells acquired in vitro and in vivo. Cancer Res 60(7):2056-2062

Holter J, Wakui E, Tazawa H, Treuter E, Gustafsson JA 2001 Regulation of glucocorticoid receptor activity by 14-3-3-dependent intracellular relocalization of the corepressor RIP140. Mol Endocrinol 15:501-511

Horwitz KB, Jackson TA, Bain DL, Richer JK, Takimoto GS, Tung L 1996 Nuclear receptor coactivators and corepressors. Mol Endocrinol 10:1167-1177

Housley PR, Sanchez ER, Westphal HM, Beato M, Pratt WB 1985 The molybdate-stabilized L-cell glucocorticoid receptor isolated by affinity chromatography or with a monoclonal antibody is associated with $90-92-\mathrm{kDa}$ nonsteroid-binding phosphoprotein. J Biol Chem 260:13810-817

Ivarie RD, O'Farrell, PH 1978 The glucocorticoid domain: steroid-mediated changes in the rate of synthesis of rat hepatoma proteins. Cell 13:41-55

Johnson BH, Ayala-Torres S, Chan LN, El-Naghy MS, Thompson EB 1997 Glucocorticoid/ oxysterol-induced DNA lysis in human leukemic cells. J Steroid Biochem Mol Biol 61:35-45

Johnson DM, Newby RF, Bourgeois S 1984 Membrane permeability as a determinant of dexamethasone resistance in murine thymoma cells. Cancer Res 44(6):2435-2440

Kaspers GJ, Pieters R, Klumper E, De Waal, FC, Veerman, AJ 1994 Glucocorticoid resistance in childhood leukemia. Leuk Lymphoma 13(3-4):198-201

Mann CL, Hughes FM Jr, Cidlowski JA 2000 Delineation of the signaling pathways involved in glucocorticoid-induced and spontaneous apoptosis of rat thymocytes. Endocrinology 141(2): $528-538$

Martinez-Climent JA 1997 Molecular cytogenetics of childhood hematological malignancies. Leukemia 11(12):1999-2021 
McKenna NJ, Lanz RB, O’Malley BW 1999 Nuclear receptor coregulators: cellular and molecular biology. Endocr Rev 20:321-344

Medh RD, Saeed MF, Johnson BH, Thompson EB 1998 Resistance of human leukemic CEM-C1 cells is overcome by synergism between glucocorticoid and protein kinase A pathways: correlation with c-myc suppression. Cancer Res 58:3684-3693

Medh RD, Wang A, Zhou F, Thompson EB 2001 Constitutive expression of ectopic $c$-myc delays glucocorticoid-evoked apoptosis of human leukemic CEM-C7 cells. Oncogene 20:46294639

Miesfeld R, Godowski PJ, Maler BA, Yamamoto KR 1987 Glucocorticoid receptor mutants that define a small region sufficient for enhancer activation. Science 236(4800):423-427

Migliorati G, Nicoletti I, D’Adamio F, Spreca A, Pagliacci C, Riccardi C 1994 Dexamethasone induces apoptosis in mouse natural killer cells and cyclotoxic T lymphocytes. Immunology 81(1):21-26

Miller AL, Johnson BH, Medh RD, Townsend CM Jr, Thompson EB 2002 Glucocorticoids and polyamine inhibitors synergize to kill human leukemic CEM cells. Neoplasia 4:68-81

Moore DE, Weise K, Zawydiwski R, Thompson EB 1985 The karyotype of the glucocorticoidsensitive, lymphoblastic human T-cell line CCRF-CEM shows a unique deleted and inverted chromosome 9. Cancer Genet Cytogenet 14:89-94

Norman MR, Thompson EB 1977 Characterization of a glucocorticoid-sensitive human lymphoid cell line. Cancer Res 37:3785-3791

Obexer P, Certa U, Kofler R, Helmberg A 2001 Expression profiling of glucocorticoid-treated T-ALL cell lines: rapid repression of multiple genes involved in RNA-, protein- and nucleotide synthesis. Oncogene 20:4324-4336

O'Farrell PH, Ivarie RD 1979 The glucocorticoid domain of response: measurement of pleiotropic cellular responses by two-dimensional gel electrophoresis. Monogr Endocrinol 12:189-201

Olah E, Balog E, Kajtar R, Pagor L, Jakab Z, Kiss C 1997 Diagnostic and prognostic significance of chromosome abnormalities in childhood acute lymphoblastic leukemia. Ann NY Acad Sci $824: 8-27$

Pearse MJ, O'Bryan M, Fisicaro N, Rogers L, Murphy B, d'Apice AJ 1992 Differential expression of clusterin inducible models of apoptosis. Int Immunol 4(11):1225-1231

Pinkoski MJ, Green DR 1999 Fas ligand, death gene. Cell Death Diff 6(12):1174-1181

Pratt WB, Toft DO 1997 Steroid receptor interactions with heat shock protein and immunophilin chaperones. Endocr Rev 18:306-360

Pui CH, Crist WM 1992 Cytogenetic abnormalities in childhood acute lymphoblastic leukemia correlates with clinical features and treatment outcome. Leuk Lymphoma 7(4):259-274

Ramdas J, Harmon JM 1998 Glucocorticoid-induced apoptosis and regulation of NF-kappaB activity in human leukemic T cells. Endocrinology 139(9):813-3821

Sanchez, ER, Meshinchi S, Tienrungroz W, Schlesinger MJ, Toft DO, Pratt WB 1987 Relationship of the $90 \mathrm{kDa}$ murine heat shock protein with the untransformed and transformed states of the I cell glucocorticoid receptor. J Biol Chem 262:6986-6991

Secker-Walker LM 1990 Prognostic and biological importance of chromosome findings in acute lymphoblastic leukemia. Cancer Genet Cytogenet 49(1):1-13

Sibley CH, Tomkins GM 1974 Isolation of lymphoma cell variants resistant to killing by glucocorticoids. Cell 2:213-220

Sibley CH, Yamamoto KR 1979 Mechanism of resistance of glucocorticoids. In: Baxter JD, Rousseau GG, eds. Glucocorticoid Hormone Actions. Berlin: Springer-Verlag; 357-376

Silverstein AM, Galgniana MD, Kanelakis KC, Ranayi C, Renoire JM, Pratt WB 1999 Different regions of the immunophilin FKBP52 determine its association with the glucocorticoid receptor, hsp90, and cytoplasmic dynein. J Biol Chem 274:36980-986 
Thompson EA Jr 1991 Insensitivity to the cytolytic effects of glucocorticoids in vivo is associated with a novel "slow death" phenotype. Cancer Res 51:5544-5550

Thompson EB 1998 Special topic: apoptosis. In: Hoffman JF, De Wee P, eds. Annual Review of Physiology. Palo Alto, CA: Annual Reviews; 525-532

Thompson EB 1999 Mechanisms of T-cell apoptosis by glucocorticoids. Trends Endocrinol Metab 10(9):353-358

Thulasi R, Harbour DV, Thompson EB 1993 Suppression of $c$-myc is a critical step in glucocorticoid-induced human leukemic cell lysis. J Biol Chem 268:18306-18312

Tonko M, Ausserlechner MJ, Bernhard D, Helmberg A, Kofler R 2001 Gene expression profiles of proliferating vs. G1/G0 arrested human leukemia cells suggest a mechanism for glucocorticoid-induced apoptosis. FASEB J 15:693-699

Van den Bogert C, Dontje BH, Melis TE, Van der Veen C, Kroon AM 1988 Inhibition of mitochondrial protein synthesis influences the glucocorticoid sensitivity of lymphoid cells. Biochim Biophys Acta 972(3):302-310

Wylie AH, Beattie GJ, Hargreaves AD 1981 Chromatin changes in apoptosis. Histochem J 13(4):681-692

Wylie AH, Morris RG, Smith AI, Dunlop D 1984 Chromatin cleavage in apoptosis: association with condensed chromatin morphology and dependence on macromolecular synthesis. J Pathol 142(1):67-77

Young DA, Voris BP, Nicholson ML 1981 Cellular and biochemical actions of adrenal glucocorticoid hormones on rat thymic lymphocytes. Envir Hlth Perspect 38:89-97

Yuh YS, Thompson EB 1987 Complementation between glucocorticoid receptor and lymphocytolysis in somatic cell hybrids of two glucocorticoid-resistant human leukemic clonal cell lines. Somat Cell Mol Genet 13:33-46

Zawydiwski R, Harmon JM, Thompson EB 1983 Glucocorticoid-resistant human acute lymphoblastic leukemia cell line with functional receptor. Cancer Res 43:3865-3873

Zhou F, Medh RD, Thompson EB 2000a Glucocorticoid mediated transcriptional repression of c-myc in apoptotic human leukemic CEM cells. J Steroid Biochem Mol Biol 73:195-202

Zhou F, Medh RD, Zhang W, Ansari N, Thompson EB 2000b The delayed induction of $c$-jun in apoptotic human leukemic lymphoblasts is primarily transcriptional. J Steroid Biochem Mol Biol 75(2-3):91-99 